\title{
BMJ Open Risk of recurrent corneal erosion in patients with diabetes mellitus in Taiwan: a population-based cohort study
}

\author{
Ren-Long Jan (D) ,,2 Ming-Cheng Tai, ${ }^{3}$ Chung-Han Ho (D) , ${ }^{4,5}$ Chin-Chen Chu, ${ }^{6,7}$ \\ Jhi-Joung Wang, ${ }^{4,6}$ Sung-Huei Tseng, ${ }^{8,9}$ Yuh-Shin Chang (D) 2,9
}

To cite: Jan R-L, Tai M-C, Ho C-H, et al. Risk of recurrent corneal erosion in patients with diabetes mellitus in Taiwan: a population-based cohort study. BMJ Open 2020;10:e035933. doi:10.1136/ bmjopen-2019-035933

- Prepublication history and additional material for this paper are available online. To view these files, please visit the journal online (http://dx.doi. org/10.1136/bmjopen-2019035933).

R-LJ and M-CT contributed equally.

Received 22 November 2019 Revised 13 April 2020 Accepted 06 May 2020
Check for updates

(C) Author(s) (or their employer(s)) 2020. Re-use permitted under CC BY-NC. No commercial re-use. See rights and permissions. Published by BMJ.

For numbered affiliations see end of article.

Correspondence to Dr Yuh-Shin Chang; yuhshinchang@yahoo.com.tw

\section{ABSTRACT}

Objective To investigate the risk of recurrent corneal erosion (RCE) in patients with diabetes mellitus (DM). Design, setting and participants This retrospective, nationwide, matched cohort study included 239854 patients with DM recruited between 2003 and 2005 from the Longitudinal Cohort of Diabetes Patients database. The control group included the same number of age-matched and sex-matched patients without DM selected from the Taiwan Longitudinal Health Insurance Database, 2000. Data for each patient were collected from the index date until December 2013.

Main outcomes and measures The incidence and risk of RCE were compared between the two groups. Cox proportional hazards regression was used to calculate the HR for RCE after adjustment for potential confounders. The cumulative RCE incidence rate was calculated using Kaplan-Meier analysis.

Results In total, 1236 patients with DM and 884 controls developed RCE during the follow-up period, resulting in an incidence rate of RCE in patients with DM (5.87/10 000 person-years (PY)) higher than that in the controls $(4.23 / 10$ 000 PY). After adjustment for potential confounders, including hypertension, hyperlipidaemia, chronic renal disease and keratoconjunctivitis sicca, patients with DM were 1.35 times $(95 \% \mathrm{Cl}, 1.24$ to 1.48$)$ more likely to develop RCE than the total sample cohort.

Conclusions DM increases the risk of RCE, which is an interdisciplinary issue. Therefore, close collaboration between endocrinologists and ophthalmologists is important in managing RCE following DM.

\section{INTRODUCTION}

The increasing prevalence of diabetes mellitus (DM) is an important public health problem worldwide because of its accompanying morbidity and mortality. ${ }^{1}$ The ocular complications related to DM are leading causes of blindness and they have become general public health issues. ${ }^{2}$ While diabetic retinopathy is the most well-known ocular complication, DM-related ocular surface complications are also important health issues.
Strengths and limitations of this study

- This study is the largest population-based study that has been conducted to explore the relationship between diabetes mellitus (DM) and subsequent recurrent corneal erosion (RCE).

- The nationwide and population-based design of this study has good statistical power and risk appraisal precision, because we included a large sample of patients with DM.

- Patients with visual disturbances in Taiwan visit an ophthalmologist rather than a general practitioner, reducing the chances of misdiagnosis and selection bias in referral centres.

- The diagnosis of DM, RCE and other comorbidity disorders relied on International Classification of Diseases, Ninth Revision codes, which may lead to disease misclassification.

- Several important confounding factors including ocular blunt trauma, corneal abrasion, postcorneal transplantation, corneal dystrophy and band keratopathy could not be evaluated, resulting in some bias in our study.

Recurrent corneal erosion (RCE) is a common clinical complaint characterised by chronic relapsing corneal disease. The most frequent clinical presentations of RCE include redness, pain, photophobia and watering of eyes. ${ }^{3}{ }^{4}$ RCE occurs frequently in patients with corneal epithelial basement membrane dystrophies and it is often associated with superficial injuries. ${ }^{4}$ Notably, the compromised ocular surface with epithelial superficial squamous cell damage related to corneal exposure could contribute to RCE development. Additionally, the most common pathophysiology of RCE is insufficient adhesion of the corneal epithelium, resulting from the reduced attachment of the epithelium to the underlying stroma through epithelial basement membrane structures. ${ }^{3}$ Ultrastructural changes in eyes with RCE include a deficient 
epithelial basement membrane and multilaminar basement membranes attached to the basal epithelial cells. ${ }^{5}$ Furthermore, reduced corneal sensory nerve innervation arising from the decreased sub-basal nerve plexus plays an important role in RCE development. ${ }^{67}$

The cornea's morphological, physiological, metabolic and clinical states are affected by the hyperglycaemia status of patients with DM. ${ }^{8}$ Decreased corneal sensitivity is also common in patients with DM. ${ }^{9}{ }^{10}$ An elevation in the corneal sensitivity threshold of the diabetic cornea might lead to abnormal neural regulation and delayed epithelial wound healing. ${ }^{11}$ Additionally, the effects of diabetes on the corneal epithelium include an irregularly thickened, multilaminated corneal epithelial basement membrane and reduced basal epithelial cell density. ${ }^{12} 13$ Furthermore, Quadrado et al reported decreased subbasal nerve density in the cornea of patients with DM. ${ }^{14}$ Considering the common pathogenic mechanisms shared by $\mathrm{DM}$ and RCE, determining whether DM is a predictor of RCE is clinically relevant. However, no large cohort studies have investigated this association between DM and RCE. Therefore, we used a nationwide populationbased data set to design a cohort study for evaluating the association between DM and RCE in Taiwan.

The current study belongs to a series of studies that we have conducted from the Longitudinal Cohort of Diabetes Patients Database that is a data subset of the National Health Insurance Research Database (NHIRD) and contains the complete medical records for a random sample of 120000 newly diagnosed patients per year from 1996 to 2013. The database was released by the Taiwan National Health Research Institute and the public applied to be part of the database through a formal application. Multiple research studies and many publications have used this database. ${ }^{1516}$ Based on the profound effects on the structures involved with ocular diseases of DM including the retinal vessels, ocular surface and optic nerve, ${ }^{17}$ it is important to investigate the association between DM and several different ocular diseases with different International Classification of Diseases, Ninth Revision, Clinical Modification (ICD-9-CM) codes, aetiologies, manifestations, comorbid conditions, pathophysiology theories and treatment modalities. Our research team planned to find any associations between DM and different ocular diseases such as retinal artery occlusion (ICD-9-CM 362.31 and 362.32), ${ }^{15}$ retinal vein occlusion (ICD-9-CM 362.35 and 362.36), corneal ulcer (ICD-9-CM 370.0, excluding 370.07), ${ }^{16}$ RCE (ICD-9-CM 371.42) and ischaemic optic neuropathy (ICD-9-CM 377.41, excluding 446.5), and it has attempted to explain the different pathophysiological reasons for these associations.

\section{METHODS}

\section{Database}

In Taiwan, National Health Insurance (NHI) scheme has been launched to provide all residents with extensive medical care coverage since 1 March 1995. After 2007, the scheme had enrolled 22.60 million individuals and comprised $>98 \%$ of the total Taiwanese population. The data in this cohort study were obtained from the Taiwan NHIRD, which supplies information regarding patient sex, date of birth, admission and discharge dates and enciphered patient identification numbers. Besides, NHIRD includes prescriptions details, costs covered and paid by $\mathrm{NHI}$ and the diagnoses and procedure codes based on ICD-9-CM. The requirement for informed consent was waived because no identifiable personal information could be analysed using the data sets from the database.

\section{Selection of patients and variables}

Two study groups were enrolled in this retrospective cohort study, both recruited during 2003-2005: a newonset DM group and a matched non-DM control group. In total, 239854 patients with DM (ICD-9-CM code 250) diagnosed between 1 January 2003 and 31 December 2005 were included. They were selected from the Longitudinal Cohort of Diabetes Patients Database, which is a data subset of the NHIRD and contains the complete medical records of a random sample of 120000 newly diagnosed patients per year from 1996 to 2013. RCE was defined as ICD-9-CM code 361.42 diagnosed after the index date of the patient with DM. Patients with missing data, unknown sex or age $<20$ years were excluded, as were patients diagnosed with RCE before DM.

For each DM patient, one non-DM control was randomly selected from the Longitudinal Health Insurance Database of 2000, which is a subdata of the NHIRD that includes the overall claim data for 1 million beneficiaries randomly selected in 2000 . No significant differences were observed in age, sex or healthcare costs between the sample group and all NHI enrollees. The 239854 controls were matched to the patients with DM by age, sex and index date. The index date for the DM patients was the date of initial diagnosis, while that for the controls it was matched to the patient's index date. Controls diagnosed with DM or RCE before the index date were excluded. Each patient was followed up to determine the RCE incidence until death or the end of 2013, whichever was earlier. The flow chart for patient enrolment was shown in figure 1. This approach for selection of study sample sizes has been used in previous studies. ${ }^{15} 16$

To differentiate patients who developed RCE after DM, every patient was tracked from her or his index date of outpatient visit or hospitalisation until December 2013, and their demographic data were recorded (eg, sex and age). Additionally, we collected data of comorbidities, including hypertension (ICD-9-CM codes 401-405), hyperlipidaemia (ICD-9-CM code 272), chronic renal diseases (ICD-9-CM codes 582-588 except 587 and 584) and keratoconjunctivitis sicca (ICD-9-CM codes 710.2 and 370.33). We considered these severe systemic diseases as possible confounders because we proposed that hypertension, hyperlipidaemia and chronic renal disease might have associations with RCE resulting from the influence of corneal nerve fibre regeneration. ${ }^{18}$ The inclusion 


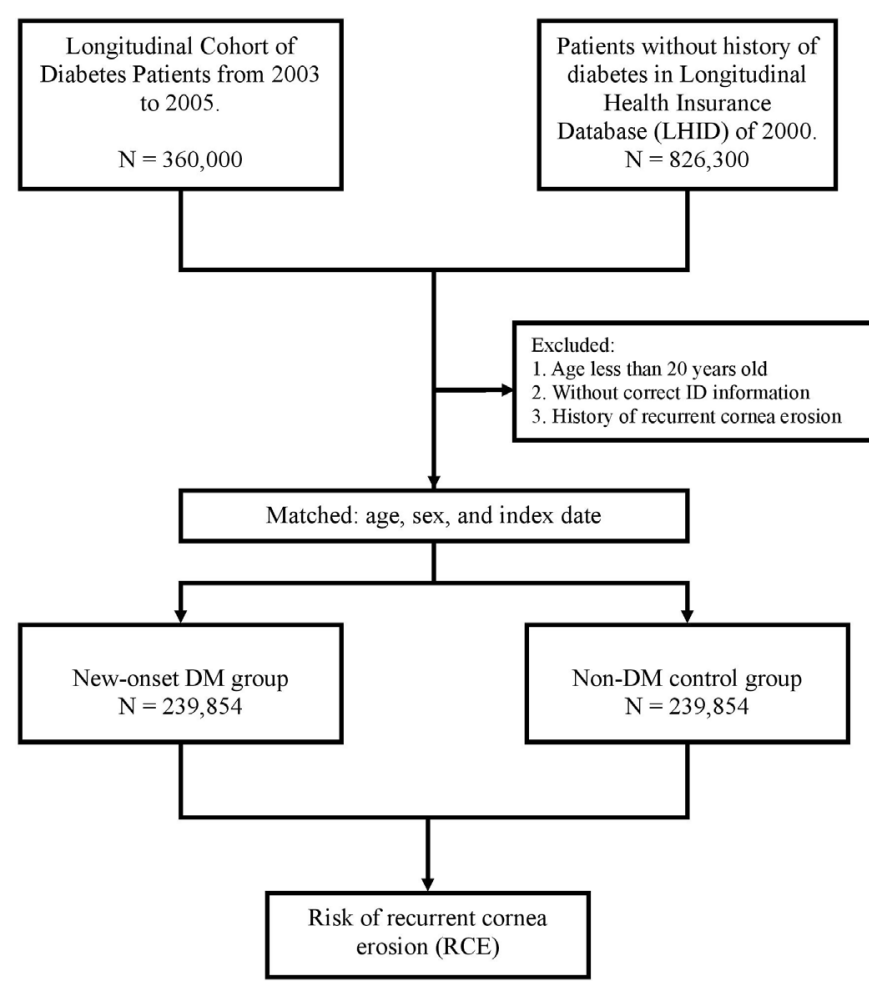

Figure 1 The flow chart for patient enrolment. DM, diabetesmellitus.

criterion for hypertension, hyperlipidaemia, chronic renal disease and keratoconjunctivitis sicca was as follows: documentation of the condition at least once in the inpatient setting or $\geq 3$ times in the ambulatory setting within 1 year before the index date. Several previous studies reported important confounders such as ocular blunt trauma (ICD-9-CM codes 921.1, 921.2, 921.3 and 921.9), corneal abrasion (ICD-9-CM code 918.1), postcorneal transplantation (order codes 85 212B, 85 213B, 85 215B, $85216 \mathrm{~B}$ and 85 217B), corneal dystrophy (ICD-9-CM code 371.5 ) and band keratopathy (ICD-9-CM code 371.43) as potential comorbidities defined by the evident criteria mentioned earlier. ${ }^{19-21}$ However, we could not evaluate whether these comorbidities were significant risk factors of RCE because of the low number of patients with these potential comorbidities or the low incidence of these potential comorbidities (online supplementary table $1 \mathrm{~S}$ ).

\section{Patient and public involvement}

It was not appropriate or possible to involve patients or the public in the design, conduct, reporting or dissemination of our research.

\section{Statistical analysis}

SAS V.9.4 for Windows (SAS Institute) was used for statistical analyses. McNemar's test was used to compare the demographic characteristics and comorbidities between the DM and non-DM control groups because of the matched-pair data. In addition, the mean age was estimated using a paired t-test, and the median follow-up time and time to RCE were determined using the Wilcoxon signed rank test. The RCE incidence was calculated as the number of patients with RCE identified during the follow-up period divided by the total number of personyears (PY) for each group by age, sex and selected comorbidities. The adjusted HRs with 95\% CIs for developing RCE were calculated using Cox proportional hazards regression analyses with the Mantel-Haenszel methods for the matched cohort design. ${ }^{22}{ }^{23}$ The baseline comorbidities were set as adjusted confounding factors, including hypertension, hyperlipidaemia, chronic renal disease and keratoconjunctivitis sicca. Kaplan-Meier analyses were performed to calculate the cumulative incidence for RCE, and log-rank tests were used to analyse the differences in the cumulative incidence curves. The proportional hazards assumption was not violated according to the assessment using Schoenfeld residuals $(\mathrm{p}=0.1501)$. Kaplan-Meier curves were generated using STATA (V.12; Stata Corp.). Statistical significance was defined as $\mathrm{p}<0.05$.

\section{RESULTS}

\section{Demographic data}

Between 2003 and 2005, 239854 DM patients and 239854 controls were recruited after excluding ineligible patients. Table 1 reveals the demographic data for the DM patients and matched controls. Data for the evaluated comorbidities are also presented in table 1 . The mean age of the DM patients and controls was 55.08 (SD, 14.86) years. Of the $239854 \mathrm{DM}$ patients, $133638(55.72 \%)$ were men and 106216 (44.28\%) women; among them, 91232 (38.04\%) were aged $20-49$ years; $85616(35.70 \%)$ were aged $50-64$ years and $63006(26.27 \%)$ were aged $\geq 65$ years. The DM group exhibited a significantly higher prevalence of comorbidities, including hypertension, hyperlipidaemia, chronic renal disease and keratoconjunctivitis sicca than did the control group. In total, 1236 patients with DM and 884 controls developed RCE, which led to a significantly higher prevalence of RCE in the DM group than in the control group. The median follow-up periods for the DM and control patients were 9.27 years (Q1-Q3, 8.43-10.12 years) and 9.33 years $(\mathrm{Q} 1-\mathrm{Q} 3,8.51-10.15$ years $)$, respectively. The median RCE development times for the DM and control patients were 4.08 years $(\mathrm{Q} 1-\mathrm{Q} 3,1.63-6.72$ years) and 4.15 years (Q1-Q3, 1.82-6.60 years), respectively. The DM group included 2643 patients $(1.10 \%)$ with type $1 \mathrm{DM}$ and 237211 patients $(98.90 \%)$ with type $2 \mathrm{DM}$.

\section{Incidence rates for RCE}

During the follow-up period, 2120 (2120/479 708, $0.442 \%$ ) patients developed RCE, with the proportion being significantly higher in the DM group (1236/239 $854,0.515 \%)$ than in the control group $(884 / 239854$, $0.369 \%$; table 2). In addition, a significant intergroup difference was observed in the RCE incidence rate (DM, 5.87/10 $000 \mathrm{PY}$; control, 4.23/10 000 PY) and adjusted HR (1.35, 95\% CI=1.24 to $1.48, \mathrm{p}<0.0001$; table 2$)$.

Patients with DM aged 50-64 years exhibited the highest RCE incidence (6.46/10 $000 \mathrm{PY})$, followed by 
Table 1 Demographic characteristics and comparison of comorbid disorders between the diabetes mellitus and control groups

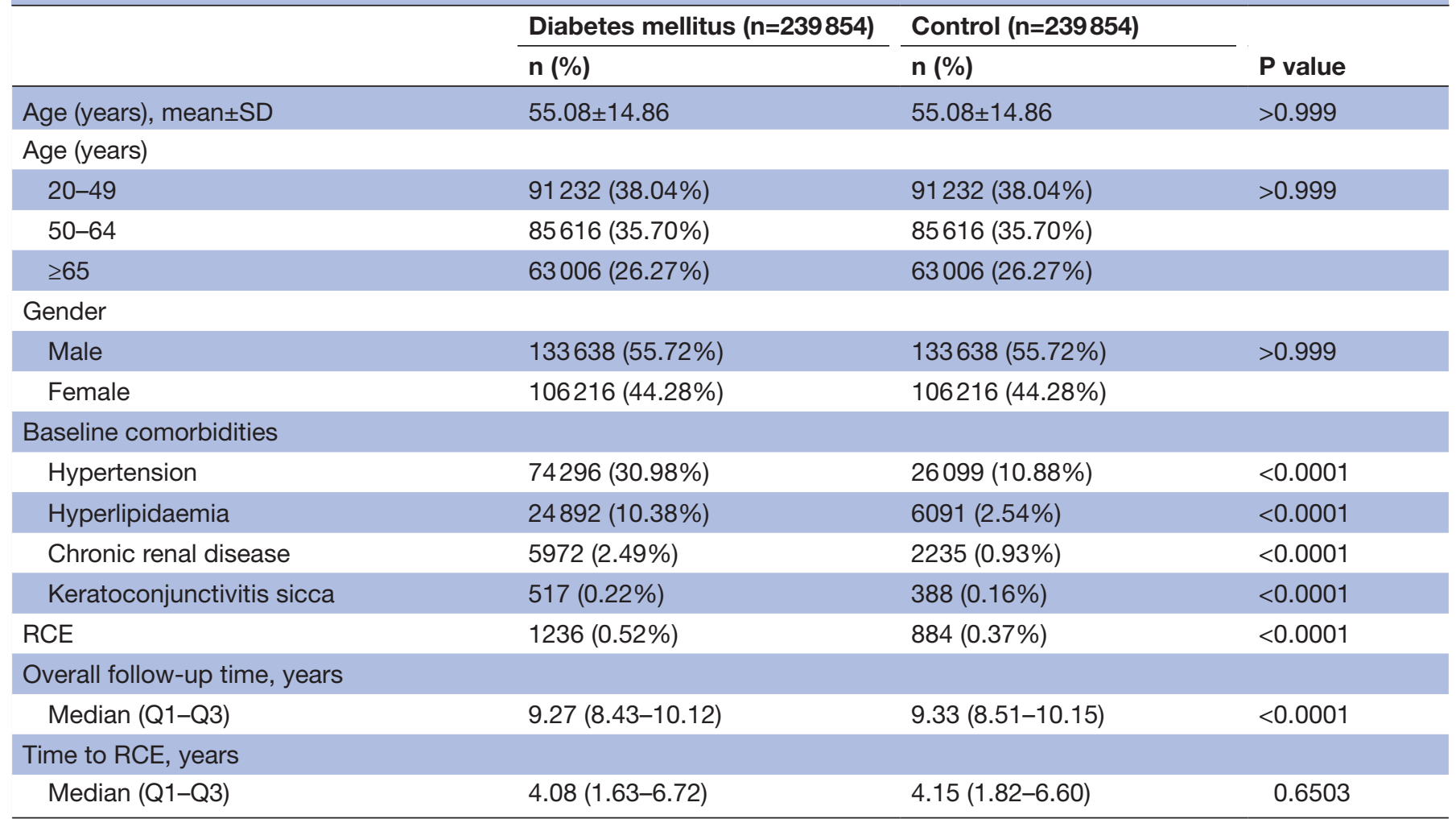

Demographic characteristics and comorbid disorders were compared between the diabetes mellitus and control groups by using McNemar's test. The mean age was estimated using paired t-test, and the median of follow-up time and time to RCE were calculated with Wilcoxon's signed rank test.

RCE, recurrent corneal erosion.

those aged $20-49$ years $(6.18 / 10000 \mathrm{PY})$ and $\geq 65$ years $(4.42 / 10000 \mathrm{PY})$. The values of adjusted HR were significantly higher for the DM patients aged 20-49 years (adjusted $\mathrm{HR}=1.61 ; 95 \% \mathrm{CI}=1.39$ to $1.85 ; \mathrm{p}<0.0001$ ) and those aged 50-64 years (adjusted $\mathrm{HR}=1.22 ; 95 \% \mathrm{CI}=1.06$ to $1.40 ; \mathrm{p}=0.0048)$ than the age-matched control groups, respectively (table 2 ).

The RCE incidence rate was 5.11/10 000 PY for DM men and 3.02/10 000 PY for male controls (adjusted $\mathrm{HR}=1.63 ; 95 \% \mathrm{CI}=1.42$ to $1.87 ; \mathrm{p}<0.0001)$. A significant difference was also observed between DM women and female controls (adjusted HR=1.17; 95\% CI=1.04 to 1.32; $\mathrm{p}=0.0081$; table 2 ).

In the DM group, the incidence rates of RCE, from the highest to the lowest, were in the order of patients with keratoconjunctivitis sicca $(28.50 / 10000 \mathrm{PY})$, hyperlipidaemia (6.59/10 $000 \mathrm{PY})$, hypertension (5.61/10 $000 \mathrm{PY})$ and chronic renal disease $(4.58 / 10000 \mathrm{PY})$. However, the adjusted HR for RCE in the patients with DM with these comorbidities did not indicate a significantly greater risk as compared with the controls.

Table 3 provides the crude and adjusted HRs for RCE during the follow-up period. After adjusting for the selected comorbidities, DM remained an independent risk factor for RCE (adjusted HR=1.35; 95\% CI=1.24 to 1.48; $\mathrm{p}<0.0001)$. Significant risk factors for RCE in both groups included hyperlipidaemia (adjusted HR, 1.19; $95 \% \mathrm{CI}=1.02$ to $1.40 ; \mathrm{p}=0.0289)$ and keratoconjunctivitis sicca (adjusted HR, $4.55 ; 95 \% \mathrm{CI}=2.89$ to 7.15 ; $\mathrm{p}<0.0001$ ). Hypertension and chronic renal disease were not independent risk factors for RCE.

Kaplan-Meier analyses disclosed higher cumulative incidence of RCE in the DM group than in the control group, and the log-rank test findings were also significant $(\mathrm{p}<0.0001$; figure 2$)$.

\section{DISCUSSION}

To our knowledge, no large-scale population-based study has previously shown the relationship between DM and subsequent RCE or investigated their pathophysiological association. Later we discussed several common pathogenic mechanisms of RCE and DM, including compromised corneal surface, corneal epithelial basement membrane abnormally and reduced sub-basal nerve plexus. Furthermore, disturbed wound healing of the cornea, the effect of epithelial stem cell abnormalities and changes in the concentrations of the growth factors and cytokines in the patients with DM may be important possible reasons for the increased RCE risk after DM. ${ }^{24}$

A compromised ocular surface, dry eye symptoms and decreased corneal sensitivity are common in DM patients, 
Table 2 Risk of RCE in the diabetes mellitus and control groups

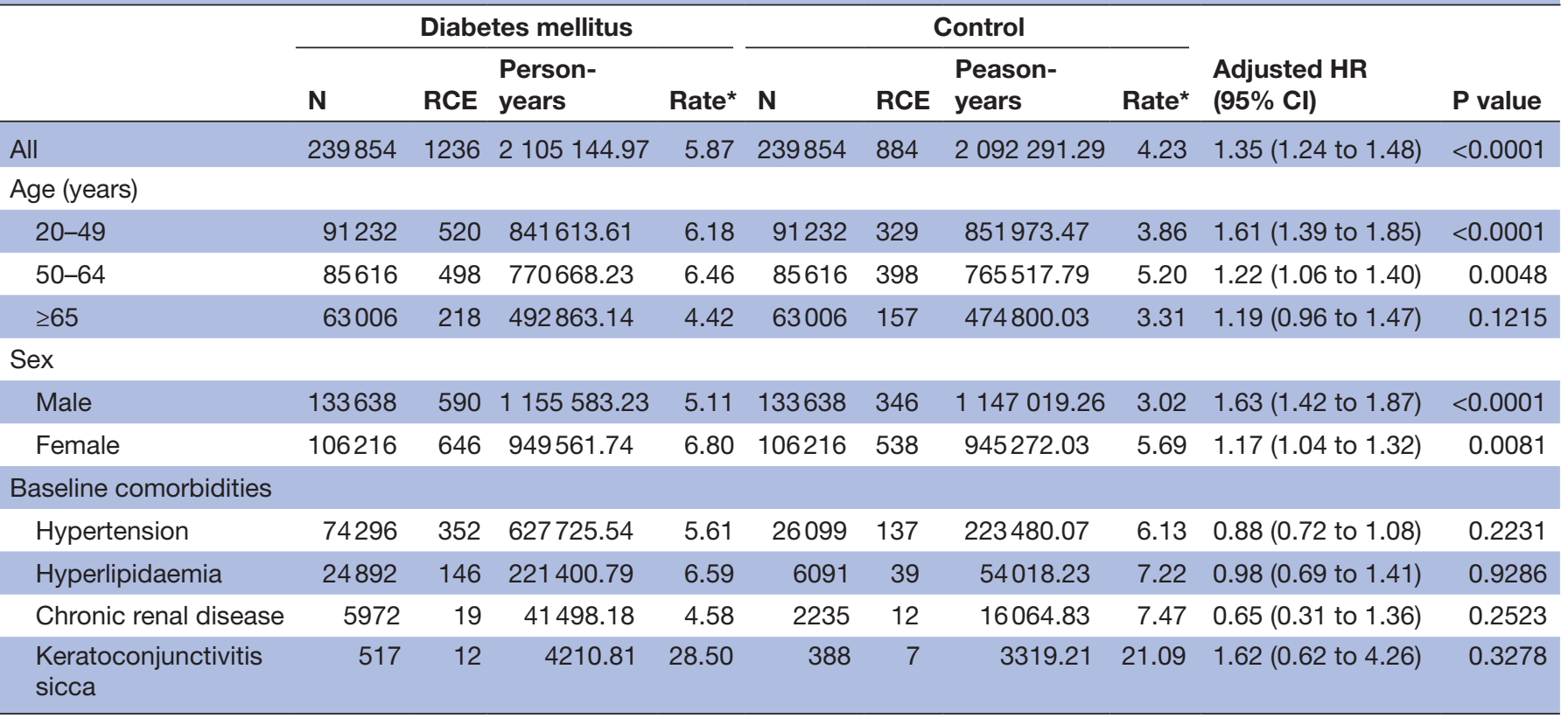

A Cox proportional hazards regression analyses with Mantel-Haenszel methods for matched cohort design was performed to calculate the adjusted HRs.

${ }^{*}$ Rate: per 10000 person-years.

$\mathrm{RCE}$, recurrent corneal erosion.

because of the reduced secretion, stability and quality of the tear film, and declined trophic effect of trigeminal sensory nerves on the cornea. ${ }^{25}$ Moreover, impaired lacrimal function and weakened wound healing capability of the corneal epithelium are frequent among DM patients and are related to higher glucose concentration in the tears. ${ }^{27} 28$ The high-glucose tear resulting from conjunctival vessel leakage in such patients harmed the microvasculature of lacrimal gland, leading to lacrimal function impairment. ${ }^{28}$ Advanced glycation end products

Table 3 Crude and adjusted HRs and 95\% Cl for recurrent corneal erosion during the follow-up period for the study cohort

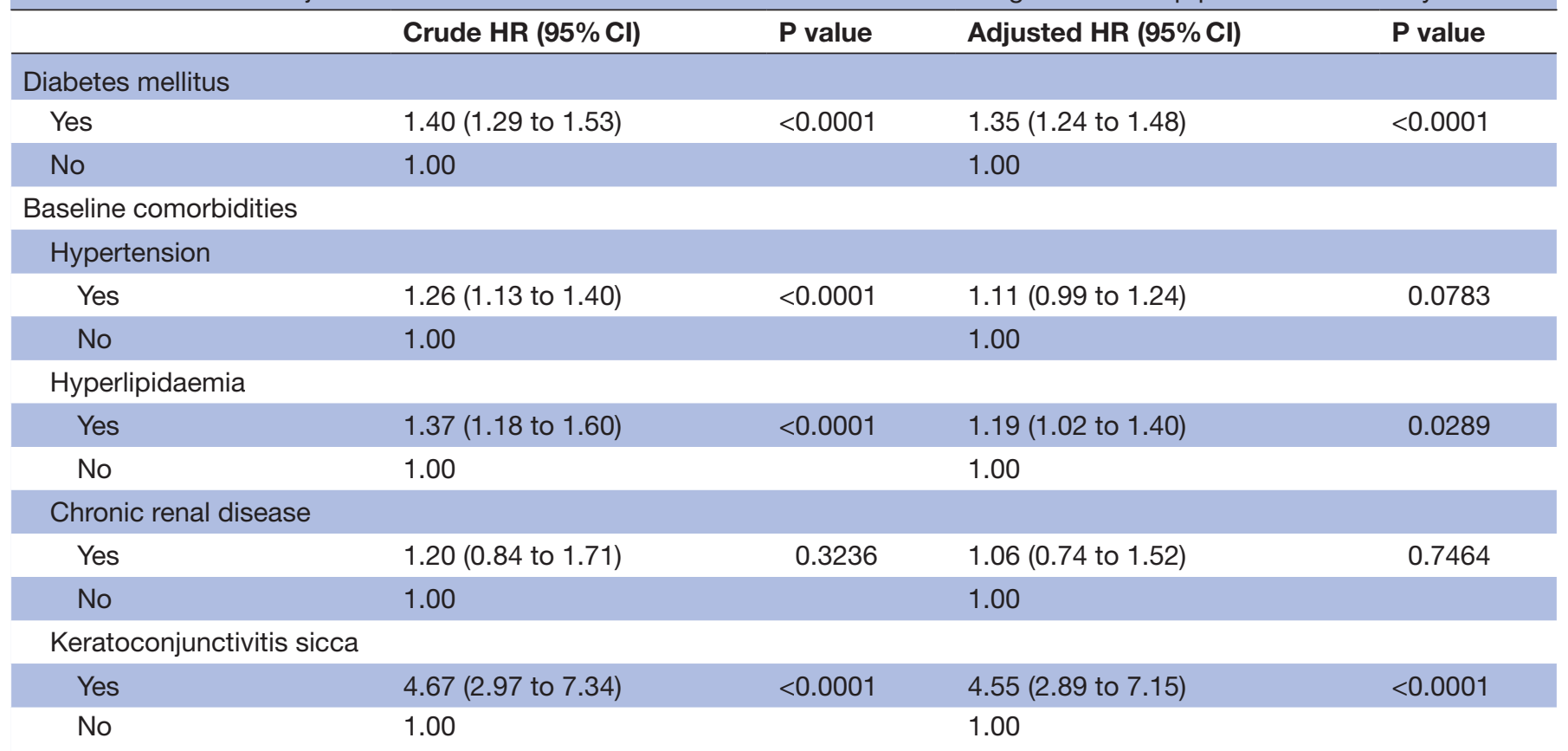

The adjusted HR for developing recurrent corneal erosion was calculated using the Cox proportional hazards regression analyses with Mantel-Haenszel methods for matched cohort design. 


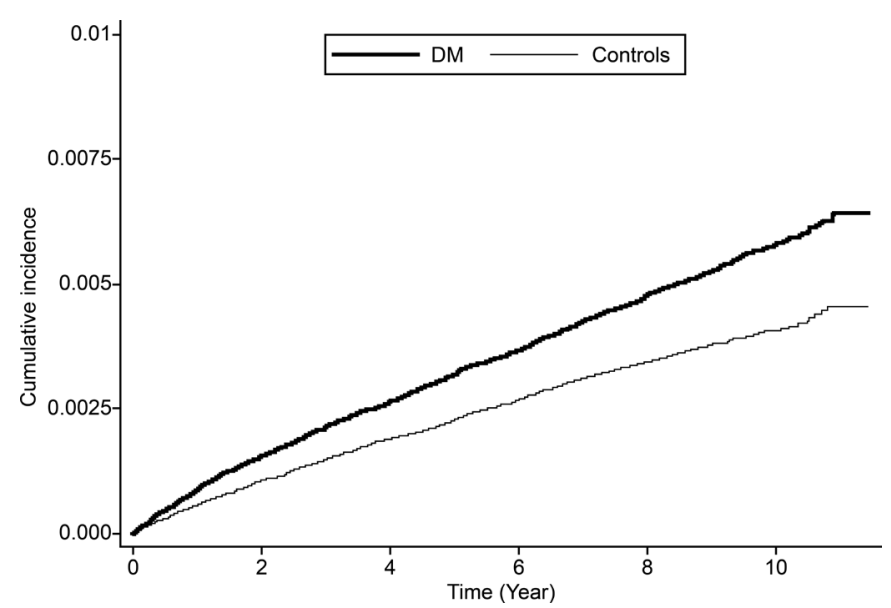

Figure 2 Cumulative incidence of recurrent corneal erosion in patients with diabetes mellitus (DM) and controls during the follow-up period.

arising from hyperglycaemia and cellular inflammatory response to oxidative stress can modify the structure of the ocular surface protein matrix, resulting in widespread tissue damage or dysfunction, and reduce the wound healing capability of the corneal epithelium..$^{25}$ Once a patient with DM develops a compromised ocular surface, it may become vulnerable to mild trauma which is the leading cause of RCE. Therefore, RCE is a frequently encountered complication of a compromised ocular surface with reduced wound healing capability.

Another possible pathogenic association between RCE and DM is increased corneal epithelial basement membrane abnormality in DM patients. Abnormalities such as irregularly thickened and multilaminated membranes have been observed in patients with DM. ${ }^{13}$ The lower basal epithelial cell density in the cornea of DM patients arising from unhealthy regulation and imbalance between cell proliferation, differentiation, migration and cell death might be related to the altered maturation of epithelial cells, epithelial degeneration, accumulation of glycogen granules and basal cell depletion. ${ }^{12}{ }^{14}$ Epithelial basement membrane abnormalities and lower basal epithelial cell density may lead to RCE development in DM patients. Consistently, the complex pathogenesis of RCE has been attributed to the defective adhesion of the corneal epithelium to the basement membrane because of a deficient epithelial basement membrane. ${ }^{34}$ Additionally, the common ultrastructural changes noted in the cornea of patients with RCE and DM include a deficient epithelial basement membrane and multilaminar basement membranes attached to the basal epithelial cells. ${ }^{5}$ These abnormalities in DM patients play an important role in RCE development.

The corneal sub-basal nerve plexus, which is capable of maintaining corneal sensitivity and normal metabolism of epithelium, is affected by DM. ${ }^{29}$ The alternations in the sub-basal nerve plexus include decreased density of sub-basal nerve, reduced nerve branching and increased nerve tortuosity. ${ }^{29}$ Recently, several studies found reduced sub-basal nerve density in the cornea of DM patients than in normal controls. ${ }^{30} 31$ Notably, the decreased corneal sensory nerve innervation resulting from the reduced sub-basal nerve plexus also plays an important role in RCE development. ${ }^{67}$

By comparing the different age groups among the DM patients, we found that the participants aged 50-64 years had the highest incidence and those aged $\geq 65$ years had the lowest incidence of RCE (table 2). This result is inconsistent with the earlier report that investigated RCE, including approximately $45 \%-64 \%$ of cases related to prior physical injury and $19 \%-29 \%$ of cases related to corneal dystrophy, and showed that the highest prevalence was observed between the age of 30 and 40 years. ${ }^{20}$ We tried to explain the inconsistency on the basis of the different RCE populations with different proportions of related mechanisms included owing to the different study designs of the studies. Our study was a cohort study aimed at investigating the RCE incidence rate in 239854DM patients with lower proportions of ocular trauma and corneal dystrophy than those in Miller's report. On the other hand, the assumption of morepronounced hyperglycemia-induced corneal changes in older patients contradicts the finding of a lower prevalence in patients aged $\geq 65$ years (table 2 ). We proposed that the death censoring might have played a role in the low incidence rate in the DM patients aged $\geq 65$ years. Among the elderly populations with DM, the proportion of patients who died before RCE development might be higher.

We found that female sex showed high incidence of RCE and is a significant risk factor in developing RCE (table 2). Female predominance is found in several reports. ${ }^{33}$ We proposed that hormonal events, such as menstruation, pregnancy and menopause, in women are aggravating factors in RCE. ${ }^{33}$ This finding could also be explained by the fact that mild trauma such as fingernail injury is the most common cause of RCE. Such injury is more common in women because a higher proportion of them have long fingernails and they have a higher probability of being scratched by a baby's finger.

In this cohort study, we assessed several comorbidities in DM patients and found that hyperlipidaemia was a significant risk factor for RCE in both groups. No study has previously shown the relationship between hyperlipidaemia and RCE. Several studies showed that hyperlipidaemia is linked to corneal lipid deposition including corneal arcus, lipid keratopathy and crystalline stromal dystrophy. ${ }^{34}$ We postulated this could be due to lipid deposition in the cornea of patients with hyperlipidaemia. In addition, we attempted to explain that the association between hyperlipidaemia and RCE might be related to the influence of hyperlipidaemia to corneal nerve fibre regeneration. ${ }^{18}$ Tavakoli et al reported that improvement in dyslipidaemia was associated with regeneration of corneal nerve fibres, which was confirmed using in vivo corneal confocal microscopy. ${ }^{18}$ Therefore, patients with DM and hyperlipidaemia should be suitably advised 
about blood lipid control to ameliorate the risk of developing RCE.

Regarding the comorbidities, keratoconjunctivitis sicca was the other significant risk factor of RCE in both groups. No study has previously shown the relationship between keratoconjunctivitis sicca and RCE. Keratoconjunctivitis sicca, a multifactorial condition and a complex ocular surface disorder, is characterised by inflammation, a loss of homeostasis of the tear film and ocular symptoms, including visual disturbance, discomfort and foreign body sensation. ${ }^{36}{ }^{37} \mathrm{~A}$ vicious cycle, in which inflammation related to hyperosmolar stress is caused by lacrimal gland destruction or degeneration, corneal sensory nerve damage and tear production reduction, that results in ocular surface damage is the most important pathophysiology of keratoconjunctivitis sicca. ${ }^{38}$ The common pathophysiological mechanisms between keratoconjunctivitis sicca and RCE may reflect the linkage of these diseases.

Our study has several strengths. Since our data set included a large sample of DM patients, this nationwide, population-based study has a high statistical power and accuracy of risk appraisal. Furthermore, the chances of misdiagnosis and selection bias in referral centres are low because patients with visual problems visit ophthalmologists rather than general practitioners in Taiwan. In addition, the database is valid because several previous studies that used the same database, including our previous study, were published. ${ }^{1516}$ At last, with a maximum longitudinal data of 10 years, the cohort study monitors RCE incidence in the DM and control groups.

This study also has some limitations. We could not confirm whether the controls had a DM history before January 1996 because the medical histories of the sampled patients could only be traced back to 1996 . In addition, several important confounding factors, including ocular blunt trauma, corneal abrasion, postcorneal transplantation, corneal dystrophy and band keratopathy, could not be evaluated as to whether these comorbidities were significant risk factors of RCE, owing to the low number of patients with these potential comorbidities (online supplementary table 1S). Finally, we could not assess whether the management of blood sugar level influences the risk of developing RCE, because the insurance claim data did not include information on the current blood sugar value or haemoglobin $\mathrm{A}_{1} \mathrm{C}$ level.

Our study showed that the risk of RCE was significantly higher in DM patients than in non-DM ones, and DM remained an independent risk factor after adjustment for hypertension, hyperlipidaemia, chronic renal disease and keratoconjunctivitis sicca in the cohort. Moreover, hyperlipidaemia and keratoconjunctivitis sicca were independent risk factors for RCE in DM patients after adjusting for other confounders. These results indicate that clinicians should educate DM patients about RCE to ensure prompt and appropriate ophthalmology visits. Close cooperation between endocrinologists and ophthalmologists is necessary to manage RCE following diagnosis of DM.

\section{Author affiliations}

${ }^{1}$ Department of Pediatrics, Chi Mei Medical Center, Liouying, Tainan, Taiwan ${ }^{2}$ Graduate Institute of Medical Science, College of Health Science, Chang Jung Christian University, Tainan, Taiwan

${ }^{3}$ Department of Ophthalmology, Tri-Service General Hospital, National Defense Medical Center, Taipei, Taiwan

${ }^{4}$ Department of Medical Research, Chi Mei Medical Center, Tainan, Taiwan ${ }^{5}$ Department of Hospital and Health Care Administration, Chia Nan University of Pharmacy and Science, Tainan, Taiwan

${ }^{6}$ Department of Anesthesiology, Chi Mei Medical Center, Tainan, Taiwan ${ }^{7}$ Department of Recreation and Health-Care Management, Chia-Nan University of Pharmacy and Science, Tainan, Taiwan

${ }^{8}$ Department of Ophthalmology, National Cheng Kung University Hospital, College of Medicine, National Cheng Kung University, Tainan, Taiwan

${ }^{9}$ Department of Ophthalmology, Chi Mei Medical Center, Tainan, Taiwan

Acknowledgements Data from the National Health Insurance Research Database were provided by the Taiwan Bureau of National Health Insurance and Department of Health.

Contributors All authors conceived the study. R-LJ, M-CT, C-HH, C-CC and Y-SC conducted the study. R-LJ, M-CT, C-HH, C-CC, S-HT and Y-SC analysed the results. J-JW provided materials. R-LJ and Y-SC wrote the article. All authors reviewed the manuscript.

Funding The authors have not declared a specific grant for this research from any funding agency in the public, commercial or not-for-profit sectors.

Competing interests None declared.

Patient and public involvement Patients and/or the public were not involved in the design, or conduct, or reporting, or dissemination plans of this research.

\section{Patient consent for publication Not required.}

Ethics approval This study was exempted from full review by the Institutional Review Board of Chi Mei Medical Center. The institutional review board approval number is 10411-E04.

Provenance and peer review Not commissioned; externally peer reviewed.

Data availability statement All data relevant to the study are included in the article or uploaded as supplementary information.

Open access This is an open access article distributed in accordance with the Creative Commons Attribution Non Commercial (CC BY-NC 4.0) license, which permits others to distribute, remix, adapt, build upon this work non-commercially, and license their derivative works on different terms, provided the original work is properly cited, appropriate credit is given, any changes made indicated, and the use is non-commercial. See: http://creativecommons.org/licenses/by-nc/4.0/.

\section{ORCID iDs}

Ren-Long Jan http://orcid.org/0000-0002-3205-7621

Chung-Han Ho http://orcid.org/0000-0001-5925-8477

Yuh-Shin Chang http://orcid.org/0000-0001-6247-5050

\section{REFERENCES}

1 Nathan DM. Diabetes: advances in diagnosis and treatment. JAMA 2015;314:1052-62.

2 Pinazo-Durán MD, Zanón-Moreno V, García-Medina JJ, et al. Eclectic ocular comorbidities and systemic diseases with eye involvement: a review. Biomed Res Int 2016;2016:1-10.

3 Ramamurthi S, Rahman MQ, Dutton GN, et al. Pathogenesis, clinical features and management of recurrent corneal erosions. Eye 2006;20:635-44.

4 Das S, Seitz B. Recurrent corneal erosion syndrome. Surv Ophthalmol 2008;53:3-15.

5 Kenyon KR. Recurrent corneal erosion: pathogenesis and therapy. Int Ophthalmol Clin 1979;19:169-95.

6 Shaheen BS, Bakir M, Jain S. Corneal nerves in health and disease. Surv Ophthalmol 2014;59:263-85.

7 Ferrari G, Ueno H, Bignami F, et al. Trigeminal stereotactic electrolysis induces dry eye in mice. Acta Ophthalmol 2013;91:e162-3.

8 Skarbez K, Priestley Y, Hoepf M, et al. Comprehensive review of the effects of diabetes on ocular health. Expert Rev Ophthalmol 2010;5:557-77. 
9 Neira-Zalentein W, Holopainen JM, Tervo TMT, et al. Corneal sensitivity in diabetic patients subjected to retinal laser photocoagulation. Invest Ophthalmol Vis Sci 2011;52:6043-9.

10 Murphy PJ, Patel S, Kong N, et al. Noninvasive assessment of corneal sensitivity in young and elderly diabetic and nondiabetic subjects. Invest Ophthalmol Vis Sci 2004;45:1737-42.

11 Rosenberg ME, Tervo TM, Immonen IJ, et al. Corneal structure and sensitivity in type 1 diabetes mellitus. Invest Ophthalmol Vis Sci 2000;41:2915-21.

12 Chang P-Y, Carrel H, Huang J-S, et al. Decreased density of corneal basal epithelium and subbasal corneal nerve bundle changes in patients with diabetic retinopathy. Am J Ophthalmol 2006;142:488-90.

13 Taylor HR, Kimsey RA. Corneal epithelial basement membrane changes in diabetes. Invest Ophthalmol Vis Sci 1981;20:548-53

14 Quadrado MJ, Popper M, Morgado AM, et al. Diabetes and corneal cell densities in humans by in vivo confocal microscopy. Cornea 2006;25:761-8.

15 Chang Y-S, Ho C-H, Chu C-C, et al. Risk of retinal artery occlusion in patients with diabetes mellitus: a retrospective large-scale cohort study. PLoS One 2018;13:e0201627.

16 Chang Y-S, Tai M-C, Ho C-H, et al. Risk of corneal ulcer in patients with diabetes mellitus: a retrospective large-scale cohort study. $\mathrm{Sci}$ Rep 2020;10:7388.

17 Sayin N, Kara N, Pekel G. Ocular complications of diabetes mellitus. World J Diabetes 2015;6:92-108.

18 Tavakoli M, Kallinikos P, Iqbal A, et al. Corneal confocal microscopy detects improvement in corneal nerve morphology with an improvement in risk factors for diabetic neuropathy. Diabet Med 2011;28:1261-7.

19 Han SB, Yang HK, Hyon JY. Influence of diabetes mellitus on anterior segment of the eye. Clin Interv Aging 2019;14:53-63.

20 Miller DD, Hasan SA, Simmons NL, et al. Recurrent corneal erosion: a comprehensive review. Clin Ophthalmol 2019;13:325-35.

21 Nanba H, Mimura T, Mizuno Y, et al. Clinical course and risk factors of recurrent corneal erosion: observational study. Medicine 2019;98:e14964.

22 Greenland S, Robins JM. Estimation of a common effect parameter from sparse follow-up data. Biometrics 1985;41:55-68.
23 Shinozaki T, Mansournia MA, Matsuyama Y. On hazard ratio estimators by proportional hazards models in matched-pair cohort studies. Emerg Themes Epidemiol 2017;14:6.

24 Ljubimov AV. Diabetic complications in the cornea. Vision Res 2017:139:138-52.

25 Alves MdeC, Carvalheira JB, Módulo CM, et al. Tear film and ocular surface changes in diabetes mellitus. Arq Bras Oftalmol 2008;71:96-103.

26 Dogru M, Katakami C, Inoue M. Tear function and ocular surface changes in noninsulin-dependent diabetes mellitus. Ophthalmology 2001;108:586-92.

27 Lane JD, Krumholz DM, Sack RA, et al. Tear glucose dynamics in diabetes mellitus. Curr Eye Res 2006;31:895-901.

28 Kaiserman I, Kaiserman N, Nakar S, et al. Dry eye in diabetic patients. Am J Ophthalmol 2005;139:498-503.

29 Kallinikos P, Berhanu M, O'Donnell C, et al. Corneal nerve tortuosity in diabetic patients with neuropathy. Invest Ophthalmol Vis Sci 2004;45:418-22.

30 Pritchard N, Edwards K, Dehghani C, et al. Longitudinal assessment of neuropathy in type 1 diabetes using novel ophthalmic markers (landmark): study design and baseline characteristics. Diabetes Res Clin Pract 2014;104:248-56.

31 Misra SL, Craig JP, Patel DV, et al. In vivo confocal microscopy of corneal nerves: an ocular biomarker for peripheral and cardiac autonomic neuropathy in type 1 diabetes mellitus. Invest Ophthalmol Vis Sci 2015;56:5060-5.

32 Reidy JJ, Paulus MP, Gona S. Recurrent erosions of the cornea: epidemiology and treatment. Cornea 2000;19:767-71.

33 Hope-Ross MW, Chell PB, Kervick GN, et al. Recurrent corneal erosion: clinical features. Eye 1994;8:373-7.

34 Patel DV. Systemic associations of corneal deposits: a review and photographic guide. Clin Exp Ophthalmol 2017;45:14-23.

35 Crispin S. Ocular lipid deposition and hyperlipoproteinaemia. Prog Retin Eye Res 2002;21:169-224.

36 Craig JP, Nelson JD, Azar DT, et al. TFOS DEWS II report executive summary. Ocul Surf 2017;15:802-12.

37 Stapleton F, Alves M, Bunya VY, et al. TFOS DEWS II epidemiology report. Ocul Surf 2017;15:334-65.

38 Bron AJ, de Paiva CS, Chauhan SK, et al. TFOS DEWS II pathophysiology report. Ocul Surf 2017;15:438-510. 